\title{
A FIXED POINT THEOREM FOR PLANE CONTINUA ${ }^{1}$
}

\author{
BY CHARLES L. HAGOPIAN ${ }^{2}$
}

\author{
Communicated by Steve Armentrout, October 9, 1970
}

\begin{abstract}
In this paper it is proved that every bounded arcwise connected plane continuum which does not separate the plane has the fixed point property.
\end{abstract}

A set $X$ is said to have the fixed point property if each continuous function $f$ on $X$ into itself leaves some point fixed (that is, there is a point $x$ belonging to $X$ such that $f(x)=x)$. The problem "Must a bounded plane continuum which does not separate the plane have the fixed point property?" has motivated a great deal of research in plane topology. K. Borsuk in 1932 proved that every Peano continuum which lies in the plane and does not separate the plane has the fixed point property [2]. Since that time, other general conditions have been found which insure that a plane continuum has this property. In 1967, H. Bell proved that every bounded plane continuum which does not separate the plane and has a hereditarily decomposable boundary has the fixed point property [1]. The following question is still outstanding. If a bounded plane continuum is arcwise connected and does not separate the plane, then must it have the fixed point property? Here an affirmative answer is given to this question by proving the following theorem. If $M$ is a bounded arcwise connected plane continuum which does not have infinitely many complementary domains, then the boundary of $M$ does not contain an indecomposable continuum.

Throughout this paper $S$ is the set of points of a simple closed surface (that is, a 2 -sphere).

The proof of the following theorem is based on techniques which are closely related to the folded complementary domain concept defined by F. Burton Jones [3, p. 173].

Theorem 1. Suppose $M$ is a continuum in $S, S-M$ does not have

AMS 1970 subject classifications. Primary 54H25, 57A05, 47H10, 55C20; Secondary $54 \mathrm{~F} 22$.

Key words and phrases. The fixed point property, arcwise connected continua, folded complementary domain, plane continua which do not separate the plane.

1 This research was partially sponsored by the Air Force Office of Scientific Research and the Sacramento State College Foundation.

2 The author gratefully acknowledges conversations about topics in this paper with Professors C. H. Papas, E. Kymala, R. Leezer, and F. B. Jones. 
infinitely many components, and $\mathrm{Bd} M$ (boundary of $M$ ) contains an indecomposable continuum $I$. Then every subcontinuum of $M$ which contains a nonempty open subset of I must contain $I$.

Proof. Suppose there exist a subcontinuum $F$ of $M$, a nonempty open subset $G$ of $I$, and a point $z$ of $I$ such that $z$ does not belong to $F$ and $G$ is contained in $F$. Let $x$ and $y$ be points of $I$ such that $x, y$, and $z$ lie on distinct composants of $I$. Let $U_{1}, U_{2}, U_{3}, \cdots$ and $V_{1}, V_{2}$, $V_{3}, \cdots$ be monotone descending sequences of circular regions in $S$ centered on and converging to $x$ and $y$ respectively such that $z$ belongs to $S-\mathrm{Cl}\left(U_{1} \cup V_{1}\right)$ (the complement of the closure of $U_{1} \cup V_{1}$ in $S$ ) and $\mathrm{Cl} U_{1} \cap \mathrm{Cl} V_{1}=\varnothing$. Suppose $M$ has exactly $\alpha$ complementary domains ( $\alpha$ is a natural number). There exists a sequence $K_{1}, K_{2}$, $K_{3}, \cdots$ of mutually exclusive continua such that for each positive integer $n$, the set $L_{n}=\bigcup_{i=1}^{2 \alpha+2} K_{(2 \alpha+2)(n-1)+i}$ is contained in $I-\left(U_{n} \cup V_{n}\right)$ and each component of $L_{n}$ meets both $\mathrm{Bd} U_{n}$ and $\mathrm{Bd} V_{n}$. Assume without loss of generality that the sequence $K_{1}, K_{2}, K_{3}, \cdots$, is such that for each positive integer $n$, there exist arc-segments $R_{1}^{n}, R_{2}^{n}, \cdots$, $R_{\alpha}^{n}$ and $E_{1}^{n}, E_{2}^{n}, \cdots, E_{\alpha}^{n}$ such that, for $i=1,2, \cdots, \alpha$,

(1) $R_{i}^{n} \subset \mathrm{Bd} U_{n}$,

(2) $E_{i}^{n} \subset \mathrm{Bd} V_{n}$,

(3) $R_{i}^{n}$ and $E_{i}^{n}$ both meet $L_{n}$ only in $K_{(2 \alpha+2)(n-1)+2 i}$ and

(4) $R_{i}^{n}$ and $E_{i}^{n}$ each have one endpoint in $K_{(2 \alpha+2)(n-1)+2 i-1}$ and the other endpoint in $K_{(2 \alpha+2)(n-1)+2 i+1}$.

The limit superior of $K_{1}, K_{2}, K_{3}, \ldots$ is a continuum in $I$ which contains $x$ and $y$. Since $x$ and $y$ do not belong to the same composant of $I$, the sequence $K_{1}, K_{2}, K_{3}$. . . must converge to $I$.

There exists a sequence of points $z_{1}, z_{2}, z_{3}, \cdots$ of $I-\mathrm{Cl}\left(U_{1} \cup V_{1}\right)$ converging to $z$ such that for each positive integer $i, z_{i}$ belongs to $K_{2 i}$. Let $Z_{1}, Z_{2}, Z_{3}, \cdots$ be a sequence of circular regions in $S-\mathrm{Cl}\left(U_{1} \cup V_{1}\right)$ such that

(1) for each positive integer $i, Z_{i}$ is centered on $z_{i}$ and has diameter less than $i^{-1}$, and

(2) if $z_{i}$ belongs to $L_{n}$ (for some integer $n$ ), then $Z_{i}$ meets only the $z_{i}$-component of $L_{n}$.

Since $I$ is in the boundary of $M$, for each positive integer $i$, there exists a point $w_{i}$ which belongs to $Z_{i} \cap(S-M)$. For each positive integer $n$, there exist points $c_{n}$ and $d_{n}$ and arc-segments $R_{n}$ and $E_{n}$ such that

(1) $c_{n}$ and $d_{n}$ are in $\left\{w_{(\alpha+1)(n-1)+i} \mid i=1,2, \cdots, \alpha+1\right\}$ and belong to the same complementary domain of $M$; 
(2) $R_{n} \cup E_{n}$ belongs to the set $\left\{R_{1}^{n} \cup E_{1}^{n}, R_{2}^{n} \cup E_{2}^{n}, \cdots, R_{\alpha}^{n} \cup E_{\alpha}^{n}\right\}$; and

(3) $R_{n} \cup E_{n} \cup I$ separates $c_{n}$ from $d_{n}$ in $S$.

It follows that for each positive integer $n$, either $R_{n} \cup I$ or $E_{n} \cup I$ must separate $c_{n}$ from $d_{n}$ in $S$ [4, Theorem 20, p. 173]. Since either $R_{n} \cup I$ separates $c_{n}$ from $d_{n}$ for infinitely many positive integers $n$ or $E_{n} \cup I$ separates $c_{n}$ from $d_{n}$ for infinitely many $n$, it may be assumed without loss of generality that $R_{n} \cup I$ separates $c_{n}$ from $d_{n}$ for each positive integer $n$. Let $W_{1}, W_{2}, W_{3}, \cdots$ be a monotone descending sequence of circular regions in $S$ centered on and converging to $z$ such that $\mathrm{Cl} W_{1} \cap \mathrm{Cl} U_{1}=\varnothing$. Assume without loss of generality that for each positive integer $n$, the points $c_{n}$ and $d_{n}$ belong to $W_{n}$.

For each positive integer $n$, define an arc $T_{n}$ and a point $x_{n}$ as follows. There exists an arc $B_{n}$ in $S-M$ from $c_{n}$ to $d_{n}$. Let $D_{n}$ denote the complementary domain of $R_{n} \cup I$ which contains $d_{n}$. Let $k_{n}$ be the first point of $B_{n} \cap\left(\operatorname{Bd} W_{n} \cap D_{n}\right)$ and let $h_{n}$ be the last point of $B_{n} \cap \mathrm{Bd} W_{n}$ which precedes $k_{n}$. Since $R_{n} \cup I$ separates $h_{n}$ from $k_{n}$ in $S$, there exists a continuum $H_{n}$ in $\left(R_{n} \cup I\right)-W_{n}$ which separates $h_{n}$ from $k_{n}$ in $S-W_{n}$ [4, Theorem 27, p. 177]. Let $T_{n}$ be the subarc of $B_{n}$ which has endpoints $h_{n}$ and $k_{n}$. Note that $T_{n} \cap \mathrm{Cl} W_{n}=\left\{h_{n}, k_{n}\right\}$. Let $Y_{1}^{n}$ and $Y_{2}^{n}$ be the mutually exclusive arc-segments in $\mathrm{Bd} W_{n}$ which have endpoints $h_{n}$ and $k_{n}$. For $i=1$ and 2, there exists a point $y_{i}^{n}$ in $H_{n} \cap Y_{i}^{n}$. The points $y_{1}^{n}$ and $y_{2}^{n}$ are contained in distinct components of $H_{n}-R_{n}$ [4, Theorem 28, p. 156]. For $i=1$ and 2, let $g_{i}^{n}$ be a point of $\mathrm{Cl} R_{n} \cap \mathrm{Cl}\left(y_{i}^{n}\right.$-component of $\left.H_{n}-R_{n}\right)$. The set ( $\theta$-curve) $T_{n} \cup \mathrm{Bd} W_{n}$ separates $g_{1}^{n}$ from $g_{2}^{n}$ in $S$. The simple closed curve $T_{n} \cup Y_{1}^{n}$ separates a point $x_{n}$ of $\left\{g_{1}^{n}, g_{2}^{n}\right\}$ from $x$ in $S$.

For each positive integer $n, T_{n}$ separates $X_{n}$, the $x_{n}$-component of $I-W_{n}$, from $A_{n}$, the $x$-component of $I-W_{n}$ in $S-W_{n}$. Since $x$ and $z$ belong to distinct composants of $I$, the sequences $X_{1}, X_{2}, X_{3}, \ldots$ and $A_{1}, A_{2}, A_{3}, \cdots$ converge to $I$. Hence there exists a positive integer $n$ such that $X_{n} \cap G \neq \varnothing, A_{n} \cap G \neq \varnothing$, and $F \cap C l W_{n}=\varnothing$. Since $T_{n} \cup$ Bd $W_{n}$ separates $X_{n} \cap G$ from $A_{n} \cap G$ and does not meet the continuum $F$, this is a contradiction. It follows that each subcontinuum of $M$ which contains a nonempty open subset of $I$ contains $I$.

THEOREM 2. Suppose $M$ is an arcwise connected continuum in $S$ and $S-M$ does not have infinitely many complementary domains. Then $\mathrm{Bd} M$ does not contain an indecomposable continuum.

Proof. Assume there exists an indecomposable continuum $I$ in Bd $M$. Let $p$ be a point of an accessible composant of $I$. Let $U_{1}, U_{2}$, 
$U_{3}, \cdots$ be a monotone sequence of circular regions in $S$ centered on and converging to $p$. Since $I$ has only countably many accessible composants and each composant of $I$ is a first category subset of $I$, the union $K$ of the inaccessible composants of $I$ is a second category subset of $I$. Since $M$ is arcwise connected, for each inaccessible composant $C$, there exists an $\operatorname{arc} A$ in $M-\{p\}$ which meets $C$ and $S-C$. Let $Q$ denote the set consisting of all such arcs. For each positive integer $n$, define $L_{n}$ to be the union of all sets $X$ such that

(1) $X$ is a component of $(I \cup \operatorname{St} Q)-U_{n}$ (StQ is the union of the elements of $Q$ ) and

(2) $X-\operatorname{Bd} U_{n}$ contains an element of $a$.

Note that for each positive integer $n$, each component of $L_{n}$ contains a triod. Hence for each $n$, the components of $L_{n}$ are countable. Since $K \subset \cup_{n=1}^{\infty}\left(L_{n}-(\mathrm{St} Q-K)\right)$, there exists an integer $n$ such that $L_{n}$ has a component $X$ with the property that $X-(\operatorname{St} Q-K)$ is somewhere dense in $I$. It follows that the closure of $X$ with respect to $M$ is a continuum in $M-\{p\}$ which contains an open subset of $I$. This is a contradiction (Theorem 1). Hence Bd $M$ does not contain an indecomposable continuum.

TheOREM 3. If $M$ is an arcwise connected bounded plane continuum which does not separate the plane, then $M$ has the fixed point property.

Proof. Theorem 2 and [1].

\section{BIBLIOGRAPHY}

1. H. Bell, On fixed point properties of plane continua, Trans. Amer. Math. Soc. 128 (1967), 539-548. MR 35 \#4888.

2. K. Borsuk, Einige Sätze über stetige Streckenbilder, Fund. Math. 18 (1932), 198-213.

3. F. B. Jones, $A$ characterization of a semi-locally-connected plane continuum, Bull. Amer. Math. Soc. 53 (1947), 170-175. MR 8, 397.

4. R. L. Moore, Foundations of point set theory, rev. ed., Amer. Math. Soc. Colloq. Publ., vol. 13, Amer. Math. Soc., Providence, R. I., 1962.

California Institute of Technology, Pasadena, California 91109

Sacramento State College, Sacramento, California 95819 